\title{
Designing strategies for the control of coccidiosis in chickens on poultry farms using modern diagnostic tools
}

This article was published in the following Dove Press journal:

Reports in Parasitology

7 January 2013

Number of times this article has been viewed

\author{
Sonja Frölich \\ Jacqueline Farhat \\ Michael Wallach \\ The iThree Institute and the School \\ of Medical and Molecular Biosciences, \\ University of Technology, Sydney, \\ Sydney, New South Wales, Australia
}

\begin{abstract}
Coccidiosis is caused by the intracellular protozoan parasite Eimeria and is a major worldwide problem in the poultry industry today. The current strategies to control the disease still rely heavily on anticoccidial drugs and on the use of shuttle programs to periodically introduce attenuated live parasites onto poultry farms. Recently, because of the improved performance of broiler chickens, farmers are able to raise chickens for market within a period of only 5-6 weeks. This short growth period enables the development of strategies for coccidiosis control either by ensuring an even exposure of chickens to parasites in the litter, inducing immunity against reinfection, or by keeping parasite numbers below a threshold level wherein only subclinical infections will occur. Therefore, the development and use of new diagnostic tools to precisely identify the Eimeria species and strain and to measure the number of oocysts in the feces and litter can be very helpful in predicting exposure and designing strategies for the control of coccidiosis in the field. The purpose of this study was to describe recently developed methods of diagnosis in terms of their sensitivity and usefulness, describe ways in which litter oocyst numbers can be quantitated, and start to develop an analytical tool that can be used for designing strategies for the control of coccidiosis. A multiplex polymerase chain reaction-based assay has been developed that enables the identification of all seven Eimeria species in a single reaction tube. Previous reports have shown it is possible to detect one oocyst using a polymerase chain reaction-based assay; however, other reports have found that under both laboratory and field conditions the minimal level of sensitivity is only 20 oocysts. In addition, the present authors' studies have found that when using spiked mixed samples, the level of detection of a contaminating Eimeria species is only $5 \%$. Therefore, it is imperative that a new method be developed with a tenfold higher level of sensitivity for field-testing. Once this test is developed and validated, it can be used to monitor the litter and assess the parameters (climate, hygiene, breed of chicken, etc) that govern coccidial outbreaks on farms.
\end{abstract}

Keywords: Eimeria, multiplex PCR, sequence-characterized amplified regions (SCARs), chronic enteritis, poultry management

\section{Introduction}

Coccidiosis is a major health problem in the poultry industry worldwide. This devastating disease causes several billion dollars of loss per annum through the clinical and subclinical effects of the disease. ${ }^{1}$ Coccidiosis is caused by the intracellular protozoan parasite Eimeria, which undergoes its life cycle in the intestinal mucosa of the infected bird. Seven Eimeria species (Eimeria maxima, Eimeria tenella, Eimeria acervulina, Eimeria necatrix, Eimeria brunetti, Eimeria mitis, and Eimeria praecox) infect chickens, leading to poor feed conversion ratios (FCRs), a loss of body weight, and increased mortality. Prophylactic anticoccidial drugs are used all over
Correspondence: Michael Wallach

The iThree Institute and the School of Medical and Molecular Biosciences, University of Technology, PO Box I23, Broadway, Sydney, New South Wales 2007, Australia Tel +6I $295 I 44082$

Fax +61295144026

Email michael.wallach@uts.edu.au
Reports in Parasitology 2013:3 I-10

(c) 2013 Frölich et al, publisher and licensee Dove Medical Press Ltd. This is an Open Access article which permits unrestricted noncommercial use, provided the original work is properly cited.
Dovepress 
the world to control the disease; however, the development of drug resistance has made it very difficult to control coccidiosis. $^{2-8}$

Eimeria parasites have a short homoxenous life cycle (4-6 days), alternating between asexual replication and sexual reproduction ultimately producing a transmissible form of the parasite, the environmentally durable oocyst, which is subsequently excreted in the feces. ${ }^{9}$ Under warm and humid environmental conditions, the excreted oocysts undergo meiosis (ie, sporulation) and become highly infectious. The seven species of Eimeria can be distinguished from one another based on the size and shape of their excreted oocysts, as well as by the location and type of intestinal lesions that are induced by an infection. For example, E. tenella produces a relatively small oocyst $(19 \times 23$ microns in size $)$ and causes bloody lesions that localize to the cecum. In contrast, E. maxima oocysts are oval in shape, much larger $(20 \times 30$ microns in size), and localize to the mid-small intestine around the diverticulum, causing dilation and thickening of the wall and producing a cheesy type of lesion.

Coccidiosis is a self-limiting disease, with chickens recovering from an infection within a few weeks. ${ }^{10}$ However, in commercial poultry houses under cramped and stressful conditions, the highly resilient oocysts build up in the feces to very large numbers (100-300,000 oocysts per gram of litter). ${ }^{11,12}$ Young broiler (meat-producing) chickens are not homogeneously exposed to the parasite for the first 1-2 weeks of growth, and as a result many of them are highly susceptible to infection at 3-5 weeks of age when there is a large number of oocysts in the litter. This results in severe clinical disease and sometimes even fatal consequences.

Live, attenuated vaccines containing a mixture of precocious strains of Eimeria oocysts have been developed over the past several years, with their main commercial use in the vaccination of breeding flocks. ${ }^{1,13-15}$ The high cost of these vaccines means their use in the treatment of broiler chickens is very expensive. To help reduce the cost of live vaccines, shuttle programs have been developed for broiler chickens in which live vaccines are rotated with coccidiostats (anticoccidial drugs). The shuttle programs have been designed in order to gain both biological and chemical control over the parasite, and to seed the litter with drugsensitive parasites, thereby lowering the selective pressure on these drugs. ${ }^{16,17}$ In order to use such a control strategy, it has become very important to have tools for determining the identity and purity of vaccine strains of Eimeria, as well as for monitoring the presence, strain type, number of oocysts, and drug sensitivity of parasites in the litter.
In addition to live vaccines, subunit and recombinant vaccines are also being used or developed in laboratories around the world. ${ }^{11,18-21}$ The research and development of these vaccines also relies on an accurate and sensitive way of determining the identity and purity of Eimeria strains used. For basic research, the incursion of contaminating Eimeria parasites in presumably purified strains can lead to invalid interpretation of data. As a result, it is crucial that very sensitive and valid tests be used for assessing strain purity, both in the field and in the laboratory.

Traditional diagnostic methods for the surveillance and control of the disease have been based on parasite size and morphology, site of infection, lesions of characteristic gross appearance, and pathogenicity. ${ }^{22}$ However, in practice these approaches can be unreliable, especially when multiple species infect chickens, as the parameters mentioned overlap among most Eimeria species. Hence, new biochemical and molecular tools have been developed over the past 2 decades for the identification of Eimeria species and strains, development of precocious strains used as attenuated vaccines, and for use in the detection of small numbers of Eimeria parasites.

In this brief review, the authors will describe the methods of diagnosis that have been recently developed in terms of their sensitivity and usefulness, describe ways in which litter oocyst numbers can be quantitated, and start to develop an analytical tool that can be used for designing strategies for the control of coccidiosis, taking into account a variety of parameters and factors.

\section{Methods for the diagnosis of Eimeria oocysts in fecal and litter samples}

Over the past 2 decades, methods used to monitor and diagnose coccidiosis outbreaks have evolved from simple but laborintensive approaches. These methods include microscopic examination of the oocysts excreted with the feces by salt flotation, microscopic observation of Eimeria parasites found in intestinal scrapings from infected chickens, ${ }^{22-24}$ and, more recently, state-of-the-art, high-throughput analysis of parasite DNA based on genetic differences between Eimeria parasites. ${ }^{25-27}$ The ultimate goal of these approaches is to be able to assess which Eimeria species and strain is involved both qualitatively and quantitatively in causing infections in the field.

Ellis and Bumstead, ${ }^{28}$ Shirley, ${ }^{29}$ and Chapman ${ }^{30}$ were the first to exploit the genetic variation within and among Eimeria species infecting domestic poultry. These pioneering studies focused on isoenzyme analysis of molecular polymorphisms 
by utilizing multilocus enzyme electrophoresis (MEE), Southern blot analysis, and chromosome karyotyping. The MEE method is based on separation of isoenzymes by size and charge; therefore, the MEE method allows identification and discrimination between Eimeria species because each species exhibits a unique electrophoretic banding profile. ${ }^{30}$ Although these approaches work and have been applied by many laboratories around the world, ${ }^{31-34}$ they have proven to be inefficient - these approaches are costly and laborious, require a large number of parasites, and fail to identify Eimeria species because of an overlap in the banding pattern, particularly in the case of a low level of enzyme polymorphism. ${ }^{35,36}$ Therefore, the MEE method cannot be used to analyze a multispecies Eimeria infection. ${ }^{37}$

In addition to the electrophoretic methods described, polymerase chain reaction (PCR) technology has been developed and applied for the identification and classification of Eimeria species infecting poultry. The main advantage of the PCR assay over the traditional biochemical and molecular approaches is the ability to selectively amplify and analyze minute amounts of parasite material. However, it is important to note that only a small proportion of the extracted DNA is subjected to the PCR reaction, and it is therefore of paramount importance that the reaction conditions are optimized in order to detect a small number of gene copies in a given sample. Nevertheless, initial PCR efforts were focused on finding variations in genome sequences or organization. Blake et $\mathrm{al}^{38}$ and Shirley and Harvey ${ }^{39}$ were some of the first to use random primers in an amplified fragment length polymorphism technique to produce restriction fragments from genomic DNA (gDNA) and construct genetic lineage maps of Eimeria species. This high-stringency fingerprinting technique is useful because it does not require previous knowledge of the genome sequence; however, it cannot be used to detect mixed-species infections, because of an overlap between the species-specific bands on electrophoretic gels.

\section{Targeted PCR on individual species}

PCR assays using defined sets of oligonucleotide primers targeting the two internal transcribed spacer (ITS) regions located between the nuclear ribosomal RNA ( $r R N A)$ genes have been developed. For example, a PCR test combined with a paper chromatography hybridization assay for Eimeria has been developed using species-specific primers for the first ITS sequences located between the $18 \mathrm{~S}$ and 5.8S rRNA genes. This test was found to be highly sensitive and could be used to differentiate between the seven species of Eimeria. ${ }^{40-42}$ However, the method required a different primer pair for each species, resulting in seven separate PCR reactions to test a given sample, and was therefore deemed labor-intensive and time-consuming. Additionally, because this approach gave limited information about strain variation, further work was carried out to develop a test based on the use of the second ITS region. ${ }^{25,27,43-45}$ Universal probes for family (Eimeriidae) and genus (Eimeria) 28S rRNA (at the $3^{\prime}$ end) and 5.8S rRNA (at the $5^{\prime}$ end) sequences respectively flanking the second ITS region enabled identification of and discrimination between all seven species. It also made it possible to detect variation within a single Eimeria species. ${ }^{44,45}$ Therefore, in addition to abolishing the need for isotopes, electrophoretic gels, and monospecific reference controls, the major advantage of this novel test was the ability to detect all seven Eimeria species and their genetic variants in a single PCR reaction. These universal primers were validated in testing two Eimeria species concurrently as well as field samples, and it was concluded by the authors that these tests are accurate, less laborious than previous tests, and cheap enough to be used for testing field samples in routine diagnostic laboratories. Although these approaches have been used as surveillance and monitoring tools because they provide valuable insights into proportions of circulating Eimeria species on broiler farms, ${ }^{43}$ they give limited information about the number of circulating oocysts at any one time.

\section{Multiplex PCR}

A few research groups have found that by using single short random primers to amplify polymorphic DNA (ie, random amplification of polymorphic DNA) they were able to identify and discriminate between Eimeria species. ${ }^{33,46,47}$ Like the amplified fragment length polymorphism approach, this type of polymerase chain reaction (random amplification of polymorphic DNA) is simple, requires no previous knowledge about the target genome sequence, and can be used to amplify minute amounts of the target template. However, because of the use of low stringency conditions (ie, low annealing temperature), this assay suffers from low reproducibility and specificity and cannot be used for mixed-species identification. To circumvent this problem, Fernandez and colleagues ${ }^{35}$ have purified and cloned species-specific bands excised from the electrophoretic gels in order to characterize their sequences. Novel primers flanking the sequence-characterized amplified regions (SCARs) have been proven to be species-specific and extremely sensitive (ie, detection threshold, 1-5 pg of target DNA) for the easy detection and differentiation between Eimeria species based on their specific migration patterns. While this assay does not provide information on genetic 
variation (which would require sequence analysis), it has been of practical use in quantification and the simultaneous detection and discrimination of the seven species of Eimeria using a single reaction tube. ${ }^{35}$

\section{Optimization of the SCAR-based multiplex PCR assay}

The present authors have employed the SCAR-based multiplex PCR assay to analyze E. maxima and E. tenella laboratory strains that have been maintained by continuous passage through 4-week-old Australorp chickens in the authors' laboratory. In addition to traditional approaches (ie, oocyst size, morphology, site of infection, and pathology), the two species were routinely identified as E. maxima and E. tenella by using the multiplex assay containing primer pairs specific for the two Eimeria species. Occasionally, microscopic examination of oocysts purified from the feces of birds infected with E. maxima showed the presence of autofluorescing, smaller contaminating oocysts, presumably E. tenella in our E. maxima oocyst stock (see Figure 1A and B). In order to confirm the presence of these two species in the authors' purified E. maxima lab strain, multiplex PCR using all seven primer pairs was performed according to methods described previously. ${ }^{35}$ Briefly, freshly shed oocysts were washed, ${ }^{48}$ cracked open by sonication, and gDNA purified and eluted using a PureLink Genomic DNA Mini Kit (Invitrogen, Carlsbad, CA, USA). Both gDNA from positive control samples of E. maxima, E. tenella, and E. acervulina and gDNA from freshly harvested oocysts were then PCR amplified at an annealing temperature of $65^{\circ} \mathrm{C}$. The identity of amplicons was analyzed by agarose gel electrophoresis (Figure 2). Under these thermocycling conditions, a single 272 base pair (bp) band was detected for E. maxima and a single band migrating at $539 \mathrm{bp}$ for E. tenella (Figure 2A). These results indicate that the strains were pure, conflicting with the microscopy results, which showed the presence of small contaminating oocysts in the authors' freshly harvested E. maxima stock.

These findings prompted the authors to test different parameters (magnesium chloride concentration, enzyme concentration, temperature, etc) in order to increase the level of sensitivity of the assay, with special emphasis on annealing temperature, to try to amplify DNA fragments that may be present in trace amounts. Under these optimized conditions in which the annealing temperature was lowered to $58^{\circ} \mathrm{C}$, electrophoresis of the gDNA amplified from E. maxima samples revealed two bands, a 272 bp band for E. maxima and a $539 \mathrm{bp}$ band for E. tenella, showing the presence of $E$. tenella in the authors' E. maxima stock as expected (Figure 2B). Surprisingly, in addition to the two bands seen in E. maxima oocysts, two bands migrating at $539 \mathrm{bp}$ and a $200 \mathrm{bp}$ band were seen in the authors' E. tenella gDNA (Figure 2C). This $200 \mathrm{bp}$ amplicon is the band size expected for $E$. necatrix gDNA. Therefore, an additional experiment was performed in which the primers for E. necatrix were removed from the multiplex assay and where the authors found that the $200 \mathrm{bp}$ amplicon disappeared, showing that this band was indeed E. necatrix (not shown). These results demonstrate the importance of optimizing the PCR conditions in order to be able to detect low levels of contaminating parasites.

The sensitivity of the multiplex PCR assay was then tested in two ways. In the first experiment, E. maxima and E. tenella oocyst stocks were titrated using a twofold dilution, and the samples were used to isolate and purify the gDNA. When individual and multiplex PCR reactions were performed, the minimum detectable oocyst number was found to be

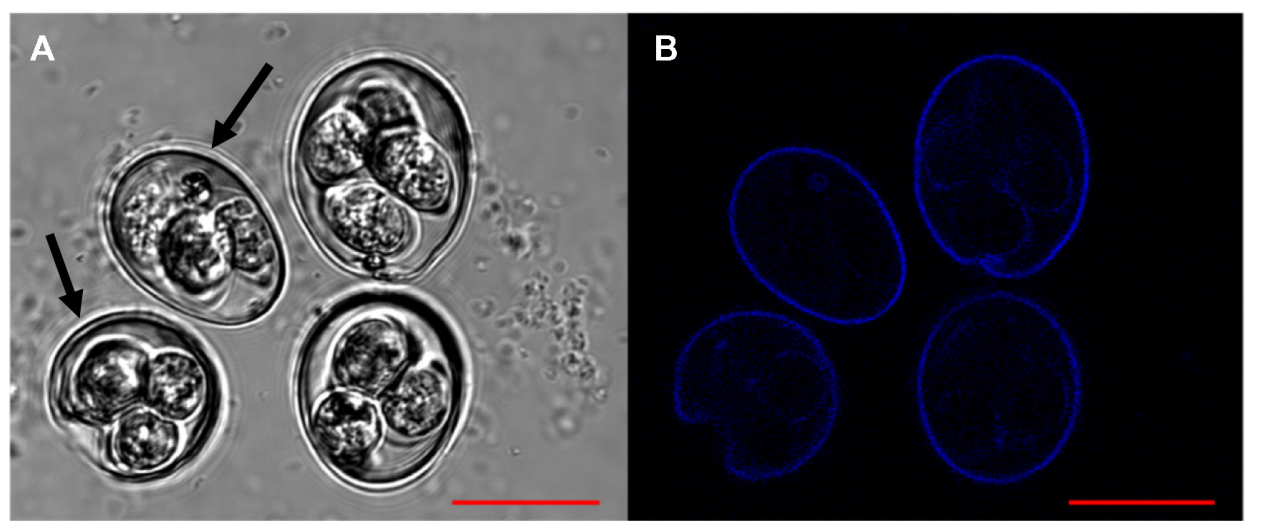

Figure I Oocysts purified from the feces collected from birds infected with Eimeria maxima: $(\mathbf{A})$ transmission detection and $(\mathbf{B})$ blue autofluorescence $($ scale bar $=20 \mu \mathrm{m})$. Notes: The presence of small ( $<20 \mu \mathrm{m}$ in diameter, arrows) oocysts in the feces. Images acquired using a Nikon scanning laser microscope (AI; Nikon Corporation, Tokyo, Japan). 

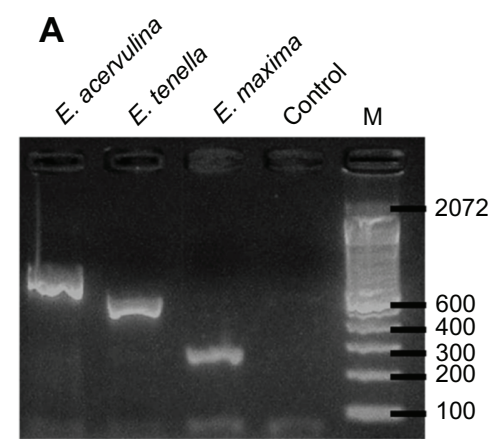

B

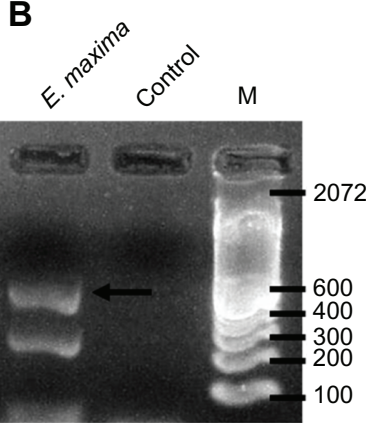

C

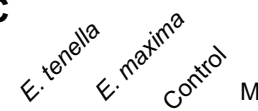

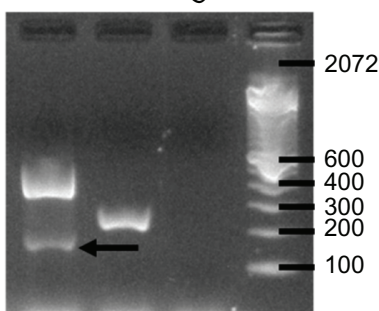

Figure 2 Agarose gel electrophoresis of multiplex polymerase chain reaction products from Eimeria tenella and Eimeria maxima laboratory stock and a control with no starting DNA: (A) a single 272 base pair (bp) E. maxima band, a single 539 bp E. tenella band, and a single $8 \mathrm{II}$ bp Eimeria acervulina band when the original protocol using $65^{\circ} \mathrm{C}$ as an annealing temperature was followed; (B) the presence of a contaminating E. tenella 539 bp band (arrow) in the E. maxima stocks was shown when the optimized annealing temperature of $58^{\circ} \mathrm{C}$ was used in the polymerase chain reaction assay; (C) contamination of $E$. tenella with Eimeria necatrix (arrow) oocysts, as revealed by the amplicon migrating at $200 \mathrm{bp}$ under optimized conditions.

Note: Molecular size markers (lane M) in bp's are indicated on the right of each panel.

10-20 for both E. maxima and E. tenella (not shown). In the second test, the ability of the multiplex PCR to detect small amounts of contaminating Eimeria species, gDNA from E. maxima were spiked with E. tenella at final concentrations of $1 \%, 5 \%$, and $10 \%$. The electrophoresis results using E. acervulina, E. tenella, and E. maxima gDNA as control samples showed that the authors were only able to detect the E. tenella band in the $10 \%$ and $5 \%$ spiked oocyst suspensions (Figure 3), showing that the detection threshold for E. tenella in the authors' laboratory is $5 \%$.

\section{Use of the PCR-based assays in the field}

In a recent publication using the SCAR-based PCR approach to individually test for the presence of Eimeria oocysts on farms in Brazil, it was found that the level of sensitivity of this test was similar to that found by the present authors, with a minimum detection level of 20 oocysts using single primer sets to test fecal samples. ${ }^{49}$ Surprisingly, if all seven primer sets were used in the multiplex PCR assay, these researchers were unable to detect any fecal oocysts excreted by 3- to 6 -week-old chickens. This result indicated that the multiplex assay is not sensitive enough to be used for field samples.

In most fecal samples taken from Brazilian farms using the single primer PCR test described, all seven species of Eimeria could be detected. However, in terms of pathology (based on lesion scoring) birds only showed signs of infection with four of the species: E. acervulina, E. tenella, E. maxima, and E. necatrix. Thus, in spite of the presence of oocysts of the other three species (E. brunetti, E. praecox,

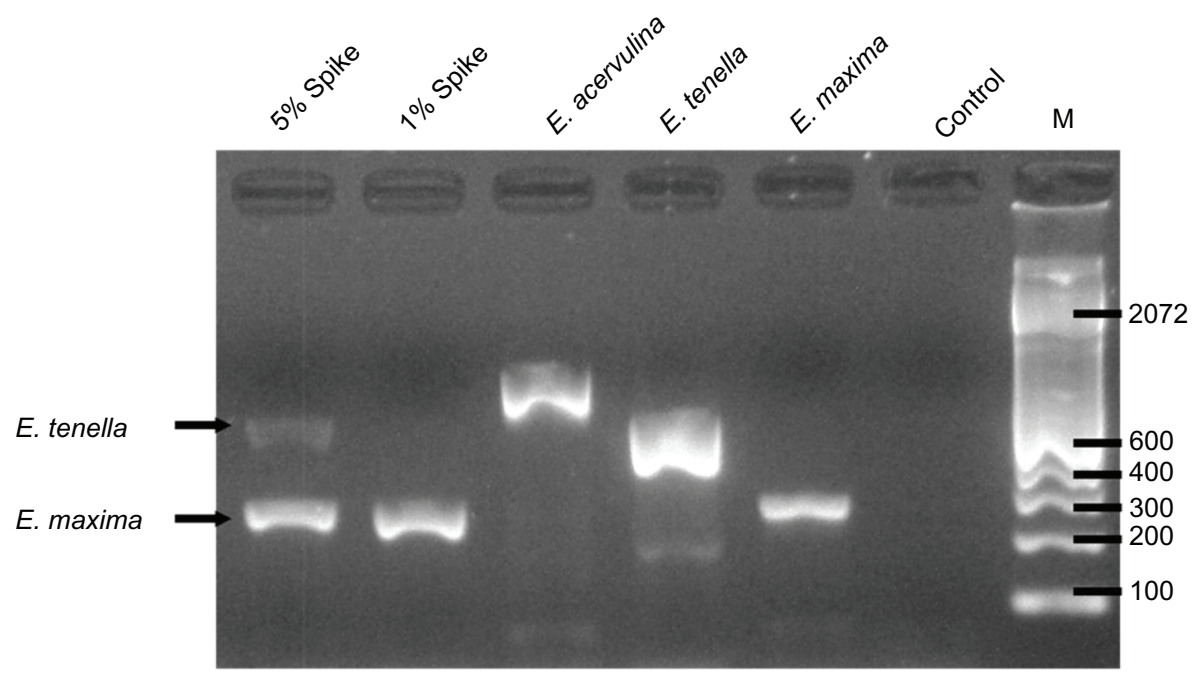

Figure 3 Sensitivity of multiplex polymerase chain reaction using $1 \%$ and $5 \%$ mixed-species suspensions.

Notes: Molecular size markers (lane M) in base pairs (bp's) and negative and positive genomic DNA controls are indicated. The gel shows a faint 539 bp Eimeria tenella band in the spiked Eimeria maxima sample. Control lanes using DNA from E. acervulina, E. tenella and E. maxima individually are shown. 
and E. mitis), no apparent pathology was induced by those species. ${ }^{49}$ Therefore, the question that needs to be asked is, why are some species and strains of Eimeria so pathogenic while others are not, and can the existing or new diagnostic tools currently being developed be used to help predict the timing and nature of outbreaks on poultry farms in order to assist veterinarians in managing coccidiosis?

\section{Improving the PCR assay}

In order to achieve a higher level of sensitivity using PCR to be able to detect very small numbers of oocysts under field conditions, improvements need to be made to the way in which fecal samples are collected on farms, the way in which oocysts are purified out of feces, the way in which DNA is extracted from oocysts, and, finally, in the PCR method itself. Starting with sample collection, it would be best to put a group of 20-30 chickens into a cage and collect fresh feces for a period of around 4-5 hours. The feces would then be processed by published methods using salt flotation, ${ }^{50}$ except the sample would be spiked with a known amount of a single pure Eimeria species to act as a carrier for processing a very small number of unknown oocysts. DNA would then be extracted and purified from both field samples as well as a control sample of DNA from the Eimeria species used to spike the sample. The PCR test would best be carried out using single or double primer pairs, and a nested PCR would be developed to maximize sensitivity. The intensity of the control spiked Eimeria band would then be used as an internal quality control sample for the assay. It is also noteworthy that only a small proportion of the DNA extracted from the oocysts is subjected to the PCR reaction. For small sample volumes containing minute amounts of gDNA or a low number of gene copies, this becomes a limiting factor. Therefore, scaling up the volume in a single reaction tube may help to ensure small amounts of DNA and gene copies are detectable by the PCR assay. Hopefully, using this suggested method, the level of sensitivity could reach one oocyst of a given Eimeria species per sample or $<1 \%$ of a mixed oocyst sample.

\section{Coccidiosis in the field: what are the key factors that promote parasite transmission, virulence, and pathogenicity?}

In order to employ the diagnostic tools described earlier to monitor the situation in the field, one first needs to understand what occurs when chickens are raised in very large numbers for commercial production and the effect of environmental conditions on parasite transmission and disease progression. To begin with, the normal picture one observes in the field is that during the first 2-3 weeks of chicken growth there are very low numbers of oocysts that cannot even be detected in litter samples by conventional means. Starting at 3-4 weeks of age there is a rapid build-up in numbers, peaking 1 week later and followed by a rapid decline in litter oocyst counts. This picture is seen whether the farm management system is based on the use of new or recycled litter. ${ }^{11,12}$ In addition, farmers will provide a 3- to 4-week break period prior to bringing in the next flock of chickens. This allows the number of oocysts to reduce to very low numbers at the start of each grow-out period.

In terms of management practice, when chicks are first brought into a farm they are often kept in a limited area of the chicken house in order to maintain the high temperature needed at that early age. Once the chicks reach 1 week of age they are dispersed throughout the chicken house. Today, because of the improvements in chicken breeding and the nutrition provided by chicken feed, the FCRs have enabled farmers to complete a grow-out within 5-6 weeks. In Europe in particular, where a small chicken is very popular for consumers, chickens are often slaughtered at 5 weeks of age (Cornish hens). By that time the chicken will weigh over $2 \mathrm{~kg}$, with a FCR often as low as 1.5-1.6. This is compared with the FCRs of 2 or greater that were the norm for 20 years prior to these improvements. Thus, the chicken farming of today is extremely efficient, and, in spite of the high cost of feed, these improvements have enabled farmers to enjoy better profitability.

Coccidiosis is, of course, a major problem when farmers are pushing chickens to grow so rapidly. Therefore, it is crucial to understand the factors that govern parasite development, transmission, and pathogenicity. Transmission of Eimeria parasites is promoted by a variety of environmental factors including heat, humidity, drug resistance, chicken density, chicken breed, immune status, and litter control (ie, wetness). Under varying climatic conditions and management practices, the transmission of the Eimeria oocyst is strongly affected. For example, in humid climates where the litter is wet, oocysts tend to spread easily and build up rapidly in broiler chickens. Chicken density will also have an effect on the rate of transmission of the parasite, where broiler chickens are usually raised at a density of 12-14 per square meter. These parameters affect the way in which chickens become exposed to the parasite and determine whether or not the chickens are naturally exposed and vaccinated against reinfection, or whether the chickens are in a highly susceptible state while the oocyst numbers rise exponentially in the litter. 
One strategy for coccidiosis control is to ensure that the litter is seeded evenly during the early start of growth, giving rise to subclinical infections in all birds and therefore enabling the chicken's natural immunity to develop. Another strategy is to ensure that there are very low numbers of oocysts for the first 3-4 weeks of growth, so that the numbers never reach a level that can cause disease up until the time the birds are slaughtered. With regard to the first strategy, it would be best to keep the chicks crowded together for as long as possible from day 1 to have a high bird density, somewhat moist conditions, and therefore good exposure to oocysts with subclinical infections. For the second strategy, it is best to spread the chickens out as much as possible, maintain dry litter, and therefore reduce oocyst transmission to a minimal level. Additionally, by employing a vaccine strategy that is aimed at reducing parasite transmission by providing maternal antibody against the sexual stages of development $\left(\mathrm{CoxAbic}^{\circledR}\right)$, it has been shown that chickens can be raised and achieve excellence performance in terms of mortality, weight gain, and FCR without the need for any in-feed anticoccidial drugs. ${ }^{11}$

The virulence and pathogenicity of Eimeria parasites also strongly affect the severity of a coccidiosis outbreak and the impact on chicken performance in the field. Parasite virulence is determined by the Eimeria species and strain type, the existence of genes that promote virulence (such as those that encode for toxins that can directly affect weight gain), and other physiological parameters. ${ }^{51}$ In addition, anticoccidial antibiotics can select for more virulent parasite strains that are drug resistant. In that regard, virulence genes need to be identified in parasite genomes that can be used as biomarkers to help predict pathogenicity of Eimeria strains and species.

The pathogenicity of Eimeria is determined both by the virulence of the parasite as well as the nature of the host-parasite relationship. In that regard, chicken breed, the immune status of the bird in terms of its susceptibility or resistance to infection, the presence of additional pathogens such as Clostridia perfringens (the causative agent of necrotic enteritis), the type of feed used, and so forth, all play a role in determining the outcome of an infection. For example, it has been observed that wheat-based feed is more likely to give rise to a problem with both coccidiosis and necrotic enteritis than corn-based feed..$^{52}$ It has also been reported that some Eimeria species and strains such as E. praecox isolated in the field can induce pathology much greater than that seen using the corresponding laboratory species or strains. ${ }^{53}$ Therefore, the approach in which attenuated oocysts present in a live vaccine are brought onto a farm to seed the litter and replace the much more virulent field strain is also an excellent biological strategy for coccidiosis control.

\section{Analytical tool for designing drug-free control strategies for coccidiosis based on good management practices in the field}

An analytical tool for designing coccidiosis control strategies can be developed that would be based on the following parameters.

1. Chicken breed and genetic resistance to infection by Eimeria

2. Chicken density (number of chickens per square meter)

3. Temperature and humidity of the environment (average over the first 3-4 weeks of growth)

4. Litter wetness (percentage of water in litter samples)

5. Virulence gene mapping and genomics (future goal)

6. Anticoccidial vaccination programs including live vaccination of young chicks and maternal immunization of breeding flocks using CoxAbic

7. Other pathogens and diseases present on the farm as assessed by veterinarians and diagnostic laboratories

8. Coccidiostats, growth-promoting antibiotics, or other drugs against diseases such as necrotic enteritis

9. Feed composition (wheat versus corn, presence of feed toxins, etc) and nutritional control

10. Number, species, and strain type of oocysts found in the litter at various time points.

Parameters 1, 2, 6, 8, and 9 are under the control of the farmer and veterinarian. Clearly, the use of chicken breeds that are naturally resistant to infection and pathogenicity by Eimeria parasites are advantageous. Chicken density can be regulated either to promote even seeding of the litter or to reduce exposure to the minimum. Vaccination programs can be designed so that in one grow-out, even seeding of the litter is aimed for through the use of live and/or transmissionblocking vaccines. New vaccine strategies to prevent infection by $C$. perfringens are currently being worked on, ${ }^{54}$ with the possibility of maternal immunization being used as a means of control for that disease as well. ${ }^{55,56}$

Feed composition can also promote better chicken performance by avoiding the use of diets that contain mostly wheat, which can exacerbate both coccidiosis and necrotic enteritis. ${ }^{47}$ In-feed coccidiostats may be used for the control of outbreaks; however, assuming the other parameters listed here are well controlled, these drugs would no longer be necessary, and should be used only in the event of a breakdown in control. 
Parameters 3, 4, and 7 are not under the control of the farmer or veterinarian. Under humid and hot environmental conditions where litter can be very wet, it would probably make the most sense to aim for an even seeding of the litter from an early age. In dry climates with good litter management the opposite would be true.

Parameter 5 is a future goal in which gene mapping and genomic sequencing of Eimeria parasites can be employed to help predict the virulence of Eimeria species and strains appearing in the field. As the technical capability to rapidly sequence Eimeria genomes improves further, this goal may begin to be achieved within the next few years.

In terms of parameter 10, the new methods of parasite identification and quantification described here can be employed, such as the multiplex SCAR-based PCR. The goal would be to be able to identify all Eimeria species in the early growth period using a PCR-based detection method that is sensitive, quick, and cost-effective. In a situation in which the goal is a rapid and even spread of the parasite throughout the chicken house, then this method can be used to ensure that this has indeed occurred and that the numbers are within an acceptable range. On the other hand, when the goal is maintaining low numbers of oocysts in the litter, the multiplex PCR can be employed to monitor the litter and decide whether or not coccidiostats are even required in the feed. If the result is that the oocyst numbers are extremely low, it is likely that the farmer can raise the chickens without any anticoccidial medication.

By carrying out field studies over a long period of time, all of the parameters described here could then be monitored, quantitated, and used to develop a set of mathematical formulas to help predict outbreaks of coccidiosis. Such an analytical tool could then be used by veterinarians to set policies that would determine when chicken flocks need to be medicated, vaccinated with live or transmission-blocking vaccines, or left without any treatment. This would greatly benefit the chicken industry as well as reduce dependence on antimicrobial drugs.

\section{Conclusion}

Over the past decade new PCR-based methods have been developed for the detection and characterization of Eimeria species in fecal samples. Based on published results together with the authors' own data, the level of sensitivity using a single or multiplex PCR assay is 20-50 oocysts per sample, which translates into around 5\%-10\% of oocysts in a mixed sample. This level of detection is good; however, in order to use this method for predicting outbreaks of coccidiosis under field conditions, a higher level of sensitivity is required in order to detect Eimeria species that may represent $<1 \%$ of the mixture or when there are only a few oocysts in a fecal sample. The authors have outlined in this review a method that could begin to reach such a level of sensitivity. Furthermore, once this method is established it can be used to help understand parasite transmission, spread, virulence, and pathogenicity in the field. This type of information can then be used by veterinarians to help make strategic decisions regarding the use of coccidiostats, the use of live vaccines, or simply allowing chickens to grow out without any anticoccidial treatments. It is hoped that this work will indeed help achieve this goal and that this method may be applied to improve health and management practices on farms to reduce dependence on antimicrobial drugs in the future.

\section{Acknowledgments}

The authors would like to thank the staff at the Ernst Animal Facility, University of Technology, Sydney, for their assistance with the care of the laboratory animals. The authors also acknowledge the financial support provided through the Australian Research Council Linkage Grant in partnership with PhiBro Animal Health Corporation, Israel. Finally, the authors thank Dr Marilyn Katrib for her help in setting the PCR-based methodology.

\section{Disclosure}

The authors report no conflicts of interest in this work.

\section{References}

1. Shirley MW, Smith AL, Tomley FM. The biology of avian Eimeria with an emphasis on their control by vaccination. In: Baker JR, Muller R, Rollinson D, editors. Advances in Parasitology. Amsterdam, The Netherlands: Elsevier; 2005;60:285-330.

2. Chapman HD, Jeffers TK, Williams RB. Forty years of monensin for the control of coccidiosis in poultry. Poult Sci. 2010;89(9):1788-1801.

3. Jeffers TK. Genetic transfer of anticoccidial drug resistance in Eimeria tenella. J Parasitol. 1974;60(6):900-904.

4. Jeffers TK. Eimeria tenella: incidence, distribution, and anticoccidial drug resistance of isolants in major broiler-producing areas. Avian Dis. 1974;18(1):74-84.

5. Jeffers TK. Eimeria acervulina and E. maxima: incidence and anticoccidial drug resistance of isolants in major broiler-producing areas. Avian Dis. 1974;18(3):331-342.

6. Jeffers TK. Anticoccidial drug resistace: differences betwen Eimeria acervulina and E. tenella strains within broiler houses. Poult Sci. 1974;53(3):1009-1013.

7. Jeffers TK. Genetic recombination of precociousness and anticoccidial drug resistance in Eimeria tenella. Z Parasitenkd. 1976;50(3):251-255.

8. Williams RB. Tracing the emergence of drug-resistance in coccidia (Eimeria spp.) of commercial broiler flocks medicated with decoquinate for the first time in the United Kingdom. Vet Parasitol. 2006;135(1):1-14.

9. Hammond DM. Life cycles and development of coccidia. In: Hammond DM, Long PL, editors. The Coccidia: Eimeria, Isospora, Toxoplasma and Related Genera. Baltimore, MD: University Park Press; 1973:45-80.

10. Rose ME, Hesketh P. Immunity to coccidiosis: stages of life-cycle of Eimeria maxima which induce, and are affected by, the response of the host. Parasitology. 1976;73(1):25-37. 
11. Wallach MG, Ashash U, Michael A, Smith NC. Field application of a subunit vaccine against an enteric protozoan disease. PLoS One. 2008;3(12):e3948.

12. Williams RB. Epidemiological aspects of the use of live anticoccidial vaccines for chickens. Int J Parasitol. 1998;28(7):1089-1098.

13. Shirley MW, Lillehoj HS. The long view: a selective review of 40 years of coccidiosis research. Avian Pathol. 2012;41(2):111-121.

14. Sharman PA, Smith NC, Wallach MG, Katrib M. Chasing the golden egg: vaccination against poultry coccidiosis. Parasite Immunol. 2010;32(8):590-598.

15. Wallach M. Role of antibody in immunity and control of chicken coccidiosis. Trends Parasitol. 2010;26(8):382-387.

16. Chapman HD, Cherry TE, Danforth HD, Richards G, Shirley MW, Williams RB. Sustainable coccidiosis control in poultry production: the role of live vaccines. Int J Parasitol. 2002;32(5):617-629.

17. Tewari AK, Maharana BR. Control of poultry coccidiosis: changing trends. J Parasit Dis. 2011;35(1):10-17.

18. Ding J, Qian W, Liu Q, Liu Q. Multi-epitope recombinant vaccine induces immunoprotection against mixed infection of Eimeria spp. Parasitol Res. 2012;110(6):2297-2306.

19. Zhang L, Ma L, Liu R, et al. Eimeria tenella heat shock protein 70 enhances protection of recombinant microneme protein MIC2 subunit antigen vaccination against E. tenella challenge. Vet Parasitol. 2012;188(3-4):239-246.

20. Wallach M, Halabi A, Pillemer G, et al. Maternal immunization with gametocyte antigens as a means of providing protective immunity against Eimeria maxima in chickens. Infect Immun. 1992; 60(5):2036-2039.

21. Wallach M, Smith NC, Braun R, Eckert J. Potential control of chicken coccidiosis by maternal immunization. Parasitol Today. 1995;11(7): 262-265.

22. Long PL, Joyner LP. Problems in the identification of species of Eimeria. J Protozool. 1984;31(4):535-541.

23. Castañón CAB, Fraga JS, Fernandez S, Gruber A, Costa LF. Biological shape characterization for automatic image recognition and diagnosis of protozoan parasites of the genus Eimeria. Pattern Recogn. 2007;40(7): 1899-1910.

24. Long PL, Millard BJ, Shirley MW. Strain variations within Eimeria meleagrimitis from the turkey. Parasitology. 1977;75(2):177-182.

25. Gasser RB, Skinner R, Fadavi R, Richards G, Morris G. High-throughput capillary electrophoresis for the identification and differentiation of seven species of Eimeria from chickens. Electrophoresis. 2005;26(18): 3479-3485.

26. Gasser RB, Woods WG, Wood JM, Ashdown L, Richards G, Whithear KG. Automated, fluorescence-based approach for the specific diagnosis of chicken coccidiosis. Electrophoresis. 2001;22(16): 3546-3550.

27. Morgan JA, Morris GM, Wlodek BM, et al. Real-time polymerase chain reaction (PCR) assays for the specific detection and quantification of seven Eimeria species that cause coccidiosis in chickens. Mol Cell Probes. 2009;23(2):83-89.

28. Ellis J, Bumstead J. Eimeria species: studies using rRNA and rDNA probes. Parasitology. 1990;101(Pt 1):1-6.

29. Shirley MW. Enzyme variation in Eimeria species of the chicken. Parasitology. 1975;71(3):369-376.

30. Chapman HD. The use of enzyme electrophoresis for the identification of the species of Eimeria present in field isolates of coccidia. Parasitology. 1982;85(Pt 3):437-442.

31. Andrews R, O’Donoghue P, Adams M, Prowse S. Enzyme markers for the genetic characterization of avian Eimeria spp. Parasitol Res. 1990;76(7):627-629.

32. Andrews RH, Chilton NB. Multilocus enzyme electrophoresis: a valuable technique for providing answers to problems in parasite systematics. Int J Parasitol. 1999;29(2):213-253.

33. Johnston DA, Fernando MA. Isoenzymes of Eimeria from the domestic fowl: electrophoretic variants among species, strains and clones. Parasitol Res. 1997;83(5):464-470.
34. Thebo P, Lunden A, Uggla A, Hooshmand-Rad P. Identification of seven Eimeria species in Swedish domestic fowl. Avian Pathol. 1998;27(6):613-617.

35. Fernandez S, Pagotto AH, Furtado MM, Katsuyama AM, Madeira A, Gruber A. A multiplex PCR assay for the simultaneous detection and discrimination of the seven Eimeria species that infect domestic fowl. Parasitology. 2003;127(Pt 4):317-325.

36. Procunier JD, Fernando MA, Barta JR. Species and strain differentiation of Eimeria spp. of the domestic fowl using DNA polymorphisms amplified by arbitrary primers. Parasitol Res. 1993;79(2):98-102.

37. Shirley MW. Coccidial parasites from the chicken: discrimination of different populations of Eimeria tenella by DNA hybridisation. Res Vet Sci. 1994;57(1):10-14.

38. Blake DP, Smith AL, Shirley MW. Amplified fragment length polymorphism analyses of Eimeria spp.: an improved process for genetic studies of recombinant parasites. Parasitol Res. 2003;90(6):473-475.

39. Shirley MW, Harvey DA. A genetic linkage map of the apicomplexan protozoan parasite Eimeria tenella. Genome Res. 2000;10(10): 1587-1593.

40. Schnitzler BE, Thebo PL, Mattsson JG, Tomley FM, Shirley MW. Development of a diagnostic PCR assay for the detection and discrimination of four pathogenic Eimeria species of the chicken. Avian Pathol. 1998;27(5):490-497.

41. Schnitzler BE, Thebo PL, Tomley FM, Uggla A, Shirley MW. PCR identification of chicken Eimeria: a simplified read-out. Avian Pathol. 1999;28(1):89-93.

42. Su XZ, Heatwole VM, Wertheimer SP, et al. The large diverse gene family var encodes proteins involved in cytoadherence and antigenic variation of Plasmodium falciparum-infected erythrocytes. Cell. 1995;82(1):89-100.

43. Morris GM, Woods WG, Richards DG, Gasser RB. The application of a polymerase chain reaction (PCR)-based capillary electrophoretic technique provides detailed insights into Eimeria populations in intensive poultry establishments. Mol Cell Probes. 2007; 21(4):288-294.

44. Woods WG, Richards G, Whithear KG, Anderson GR, Jorgensen WK, Gasser RB. High-resolution electrophoretic procedures for the identification of five Eimeria species from chickens, and detection of population variation. Electrophoresis. 2000;21(17): 3558-3563.

45. Woods WG, Whithear KG, Richards DG, Anderson GR, Jorgensen WK, Gasser RB. Single-strand restriction fragment length polymorphism analysis of the second internal transcribed spacer (ribosomal DNA) for six species of Eimeria from chickens in Australia. Int J Parasitol. 2000;30(9):1019-1023.

46. Fernandez S, Katsuyama AM, Kashiwabara AY, Madeira A, Durham AM, Gruber A. Characterization of SCAR markers of Eimeria spp. of domestic fowl and construction of a public relational database (the Eimeria SCARdb). FEMS Microbiol Lett. 2004;238(1): $183-188$.

47. Fernandez S, Costa AC, Katsuyama AM, Madeira A, Gruber A. A survey of the inter- and intraspecific RAPD markers of Eimeria spp. of the domestic fowl and the development of reliable diagnostic tools. Parasitol Res. 2003;89(6):437-445.

48. Shirley MW. Eimeria and Isospora: Eimeria species and strains of chickens. In: Eckert J, Braun R, Shirley MW, Coudert P, editors. COST 89/820 Biotechnology: Guidelines on Techniques in Coccidiosis Research. Luxembourg: European Commission; 1995:1-11.

49. Carvalho FS, Wenceslau AA, Teixeira M, Albuquerque GR. Molecular diagnosis of Eimeria species affecting naturally infected Gallus gallus. Genet Mol Res. 2011;10(2):996-1005.

50. Wagenbac GE. Purification of Eimeria tenella sporozoites with glass bead columns. J Parasitol. 1969;55(4):833-838.

51. Park SS, Lillehoj HS, Allen PC, et al. Immunopathology and cytokine responses in broiler chickens coinfected with Eimeria maxima and Clostridium perfringens with the use of an animal model of necrotic enteritis. Avian Dis. 2008;52(1):14-22. 
52. Annett CB, Viste JR, Chirino-Trejo M, Classen HL, Middleton DM, Simko E. Necrotic enteritis: effect of barley, wheat and corn diets on proliferation of Clostridium perfringens type A. Avian Pathol. 2002;31(6):598-601.

53. Williams RB, Marshall RN, Pagés M, Dardi M, del Cacho E. Pathogenesis of Eimeria praecox in chickens: virulence of field strains compared with laboratory strains of E. praecox and Eimeria acervulina. Avian Pathol. 2009;38(5):359-366.

54. Lanckriet A, Timbermont L, Eeckhaut V, Haesebrouck F, Ducatelle R, Van Immerseel F. Variable protection after vaccination of broiler chickens against necrotic enteritis using supernatants of different Clostridium perfringens strains. Vaccine. 2010;28(36):5920-5923.
55. Crouch CF, Withanage GS, de Haas V, Etore F, Francis MJ. Safety and efficacy of a maternal vaccine for the passive protection of broiler chicks against necrotic enteritis. Avian Pathol. 2010;39(6):489-497.

56. Lovland A, Kaldhusdal M, Redhead K, Skjerve E, Lillehaug A. Maternal vaccination against subclinical necrotic enteritis in broilers. Avian Pathol. 2004;33(1):83-92.

\section{Publish your work in this journal}

Reports in Parasitology is international, peer-reviewed, open access journal publishing original research, reports, reviews and commentaries on all areas of parasitology. The manuscript management system is completely online and includes a very quick and fair peer-review

\section{Dovepress}

system. Visit http://www.dovepress.com/testimonials.php to read real quotes from published authors. 